\title{
Black-hole spectroscopy: testing general relativity through gravitational-wave observations
}

\author{
Olaf Dreyer ${ }^{1}$, Bernard Kelly ${ }^{2}$, Badri Krishnan ${ }^{3}$, Lee Samuel Finn ${ }^{4}$, \\ David Garrison ${ }^{5}$ and Ramon Lopez-Aleman ${ }^{6}$ \\ ${ }^{1}$ Perimeter Institute of Theoretical Physics, 35 King Street North, Waterloo, Ontario, \\ N2J 2G9, Canada \\ ${ }^{2}$ Center for Gravitational Wave Physics, Center for Gravitational Physics and Geometry and \\ Department of Physics, 104 Davey Laboratory, University Park, PA 16802, USA \\ ${ }^{3}$ Max Planck Institut für Gravitationsphysik, Am Mühlenberg 1, D-14476 Golm, Germany \\ ${ }^{4}$ Center for Gravitational Wave Physics, Center for Gravitational Physics and Geometry, \\ Department of Physics and Department of Astronomy and Astrophysics, 104 Davey Laboratory, \\ University Park, PA 16802, USA \\ ${ }^{5}$ University of Houston, Clear Lake, 2700 Bay Area Bvd, Room 3531-2, Houston, \\ TX 77058, USA \\ ${ }^{6}$ Physical Sciences Department, University of Puerto Rico, Rio Piedras Campus, Rio Piedras, \\ Puerto Rico 00931, USA \\ E-mail: odreyer@perimeterinstitute.ca,kelly@gravity.psu.edu,badkri@aei.mpg.de, \\ lsfinn@psu.edu, garrison@cl.uh.edu and rlopez@uprrp.edu
}

Received 1 September 2003

Published 5 January 2004

Online at stacks.iop.org/CQG/21/787 (DOI: 10.1088/0264-9381/21/4/003)

\begin{abstract}
Assuming that general relativity is the correct theory of gravity in the strongfield limit, can gravitational-wave observations distinguish between black holes and other compact object sources? Alternatively, can gravitationalwave observations provide a test of one of the fundamental predictions of general relativity: the no-hair theorem? Here we describe a definitive test of the hypothesis that observations of damped, sinusoidal gravitational waves originate from a black hole or, alternatively, that nature respects the general relativistic no-hair theorem. For astrophysical black holes, which have a negligible charge-to-mass ratio, the black-hole quasi-normal mode spectrum is characterized entirely by the black-hole mass and angular momentum and is unique to black holes. In a different theory of gravity, or if the observed radiation arises from a different source (e.g., a neutron star, strange matter or boson star), the spectrum will be inconsistent with that predicted for general relativistic black holes. We give a statistical characterization of the consistency between the noisy observation and the theoretical predictions of general relativity and a demonstration, through simulation, of the effectiveness of the test for strong sources.
\end{abstract}

PACS number: $04.80 . \mathrm{Cc}$ 


\section{Introduction}

The formation of a black hole (BH) is the ultimate expression of strong-field gravity. Although we lack detailed information about the gravitational radiation produced through most of the formation process, our knowledge of the near-end point affords us important insight into the nature of general relativity.

During the late stages in the aspherical formation of an astrophysical black hole the gravitational waves emitted are dominated by a set of quasi-normal modes (QNMs) [1-3]: waves with exponentially damped sinusoidal time dependence, whose frequency and damping times are characteristic of the mass and angular momentum of the black hole ${ }^{7}$. If we observe a QNM from a black hole and also know which particular normal mode we are observing, we can determine, from the mode's frequency and damping time, the black-hole mass and angular momentum $[4,5]$.

If, on the other hand, we simultaneously observe two or more QNMs from the same source and find that they are inconsistent with the spectrum predicted by general relativity in the sense that they cannot be explained by a single value of the mass and angular momentum, we may infer that we are not observing a black hole.

A different perspective is offered by future observations by the Laser Interferometer Space Antenna (LISA) [6]. LISA is expected to observe mergers of compact objects with masses in the range $10^{6}-10^{8} M_{\odot}$ [7]. In our present understanding, these compact objects can only be black holes. Thus, observations by LISA of QNMs inconsistent with black holes would also conflict with the general relativistic no-hair theorem since an inconsistency in this mass range with black-hole sources would indicate that physical scales other than mass and angular momentum were involved in the generation of the radiation.

Here we develop this observation into an experimental test of the existence of black holes or, alternatively, a test of general relativity itself. In either sense the test described here is of general relativity based on gravitational-wave observations.

Damped sinusoidal motion is ubiquitous for systems approaching equilibrium and one expects that collapse or coalescence will lead, in any theory of gravity, to some form of QNM ringing. If we observe a QNM spectrum that is inconsistent with an isolated black hole, then there are two possibilities. On the one hand, general relativity may not be the correct theory of gravity in the strong-field limit. On the other hand, general relativity may yet be correct, but we are not observing an isolated black hole approaching equilibrium ${ }^{8}$. Alternatively, we may be observing the radiation arising from a compact body that is not a black hole, for example, a neutron star [8], a boson star [9] or strange matter star [10], whose QNM spectrum will be determined by the properties and configuration of the appropriate matter fields, or a black hole carrying a previously unknown macroscopic charge (e.g., a dilaton field [11]). Thus, while no single observation may rule out general relativity, a set of observations, each of a different source, none of which is consistent with an isolated black hole, could suggest the need to consider alternative theories of gravity in the strong-field limit.

Gravitational waves have been suggested before as probes of general relativity. Eardley et al [12] proposed the first test of general relativity using gravitational-wave observations. They investigated the polarization modes of gravitational waves in various metric theories of gravity and described how to identify the polarization modes experimentally and use these

7 And electric charge, as well; however, astrophysical black holes, which are our interest here, have negligible charge-to-mass ratio.

8 In fact, the uniqueness theorems have only been proved for vacuum spacetimes and they are not true in the presence of arbitrary matter fields or radiation. Nevertheless, it would be a great surprise if the spacetime in the vicinity of a black hole is not close to Kerr in some approximate sense. 
observations to identify the spin content of dynamical gravity. The first actual test of general relativity relying on its prediction of the existence of gravitational waves was made by Taylor and Weisberg [13], who showed that the observed orbit and orbit decay of the Hulse-Taylor binary pulsar PSR B1913+16 led to a strong consistency check on the predictions of general relativity. Finn [14] proposed a different test of the spin content of dynamical gravity, based on the possibility of a space-based detector in circumsolar orbit observing the induction-zone field associated with solar oscillations. Ryan [15] has outlined how observations of the gravitational radiation from capture orbits of solar mass compact bodies about a supermassive black hole may allow the determination of certain multipole moments of the central hole, thereby testing the prediction of general relativity. More recently, Will [16] and Finn and Sutton [17, 18] have described tests of general relativity that bound the mass of the graviton, and Scharre and Will [19] and Fairhurst et al [20] have shown how gravitational-wave observations of pulsars may be used to bound the value of the Brans-Dicke coupling constant.

The preceding tests can be grouped into three different classes. One set of tests, including $[13,15,17,19]$, is based on energy conservation arguments: the observed evolution of a system or of the radiation from a system is related to the energy loss expected owing to the radiation. A second class of tests $[12,14,20]$ focuses on the observed polarization modes of the field. The third class [16] involves the frequency-dependent dispersion relationship associated with a massive graviton. The test described in this paper is of a new class, based on the unique character of the radiation spectrum associated with a disturbed black hole.

This paper is organized as follows. In section 2 we briefly describe the QNMs of a Kerr black hole and explain how the idealized observation of two or more modes in the absence of noise enables us to extract the mass and angular momentum of the black hole. Real boats, of course, rock, and section 3 generalizes the discussion to include experimental errors and describes how one can use noisy gravitational-wave observations of QNMs to test general relativity. In section 4 we provide a proof-of-principle demonstration, via numerical simulation, of the use of this method as applied to LISA observations. Section 5 investigates the observable range (and associated event rate) within which we can expect LISA to observe sources strong enough for this test to be applied. We conclude in section 6 with a summary of our main results.

\section{Ideal observations}

\subsection{Quasi-normal modes of Kerr black holes}

Following aspherical collapse to a black hole, one expects that the final spacetime can be described as the perturbation of a stationary Kerr hole. The dominant part of the gravitational waves emitted as the black hole settles down can be described as a sum over a countably infinite set of damped sinusoids, each characterized by an amplitude, phase, frequency and damping time. (At still later times, the radiation will be dominated by power-law tails arising from the backscatter of radiation off the spacetime curvature in the neighbourhood of the black hole [1-3]; however, here we are interested in the earlier, and higher amplitude, QNM ringing.) In this sub-section we review those properties of the black-hole QNM spectrum that are important for our investigation; more detailed examinations of the spectrum itself can be found in [21-26].

QNMs appear as solutions to the equations describing perturbations of a stationary blackhole spacetime, subject to the boundary conditions of no in-going radiation from infinity and no up-coming radiation from the horizon. The perturbation equations describing Schwarzschild black holes were first described by Regge and Wheeler [27] and Zerilli [28, 29]. The first 
QNM solutions to these equations were found by Vishveshwara [30]. Teukolsky found the corresponding perturbation equations for Kerr black holes [31, 32], and, with Press, first investigated their QNM solutions [33].

Gravitational-wave detectors respond to a linear combination of the radiation in the two polarization modes of the incident gravitational waves. The observable strain $h(t)$ in, for example, the arms of an interferometric detector may be written, for QNMs, in the form

$$
h(t) \simeq \operatorname{Re}\left[\sum_{\ell, m, n} A_{\ell m n} \mathrm{e}^{-\mathrm{i}\left(\omega_{n \ell m} t+\phi_{n \ell m}\right)}\right]
$$

where the summation indices characterize the particular mode, which is related to the angular dependence of the mode amplitude and phase on a sphere of constant (Boyer-Lindquist) radius about the black hole through $\ell$ and $m$, and the 'harmonic' through the index $n$ : $\ell=2,3, \ldots,|m| \leqslant \ell$ and $n=1,2 \ldots$ For the Schwarzschild geometry the symmetry is spherical, the appropriate decomposition of the metric perturbation is given by the usual spherical harmonics and modes differing only in $m$ are degenerate. For Kerr the symmetry is axisymmetric and the orthonormal decomposition of the perturbation is by spheroidal harmonics [32]. The amplitudes $A_{n \ell m}$ and phases $\phi_{n \ell m}$ depend on the initial conditions and the relative orientation of the detector and the source; however, the complex frequency $\omega_{n \ell m}$ depends only on the intrinsic parameters of the underlying black hole, i.e., its mass $M$ and angular momentum $a M^{2}$. (We assume that the black hole carries no significant electric charge.)

For fixed $a$ the complex frequency $\omega_{n \ell m}$ scales as $M^{-1}$; thus, we define the dimensionless frequency $\Omega_{n \ell m}$,

$$
\Omega_{n \ell m}:=M \omega_{n \ell m}:=\left(2 \pi F_{n \ell m}+\frac{\mathrm{i}}{T_{n \ell m}}\right)
$$

where $F_{n \ell m}$ and $T_{n \ell m}$ are the real dimensionless frequency and damping time of the modes, respectively. The corresponding physical frequency $f_{n \ell m}$ and damping time $\tau_{n \ell m}$ are given by

$$
\omega_{n \ell m}=2 \pi f_{n \ell m}+\mathrm{i} / \tau_{n \ell m}=2 \pi F_{n \ell m} / M+\mathrm{i} /\left(M T_{n \ell m}\right) .
$$

(We use geometrical units with $G=1$ and $c=1$.) The dimensionless $\Omega_{n \ell m}$ (or $F_{n \ell m}$ and $T_{n \ell m}$ ) depend (for astrophysically relevant black holes) only on the also dimensionless black-hole angular momentum parameter $a$. Figure 1 shows $\Omega_{n \ell m}$ as a function of $a$ for $n=1,2$ and $\ell=2,3$, and $|m| \leqslant \ell$.

\subsection{From quasi-normal modes to testing relativity}

If we observe only one mode, characterized by its complex frequency $\omega$ (cf equation (3)), what can we say about the underlying black hole?

Corresponding to the observed $\omega$ is the line $\Omega=M \omega, M \in \mathbb{R}_{\geqslant 0}$, in the dimensionless $\Omega$ plane (cf equation (2)). Such a line is shown in figure 1 . This line will intersect some subset of the family of $\Omega_{n \ell m}$ curves, characteristic of black-hole normal modes. Each intersection corresponds to a black-hole mass $M$, angular momentum parameter $a$ and mode $n \ell m$ consistent with the observed $\omega$. Knowing only $f$ and $\tau$, then, we cannot uniquely identify the black-hole mass and angular momentum, but we can reduce the possibilities to a (possibly countably infinite) set of $(a, M)$ pairs. If we knew $n \ell m$ as well, we would know $a$ and $M$ exactly.

Now suppose that we observe two modes from the same black hole, each characterized by its own frequency and damping time. Figure 2(a) shows, in schematic form, the line $M \omega$ for each of the two modes (denoted by + and $\times$ ) and their intersection with several different $\Omega_{n \ell m}$ 


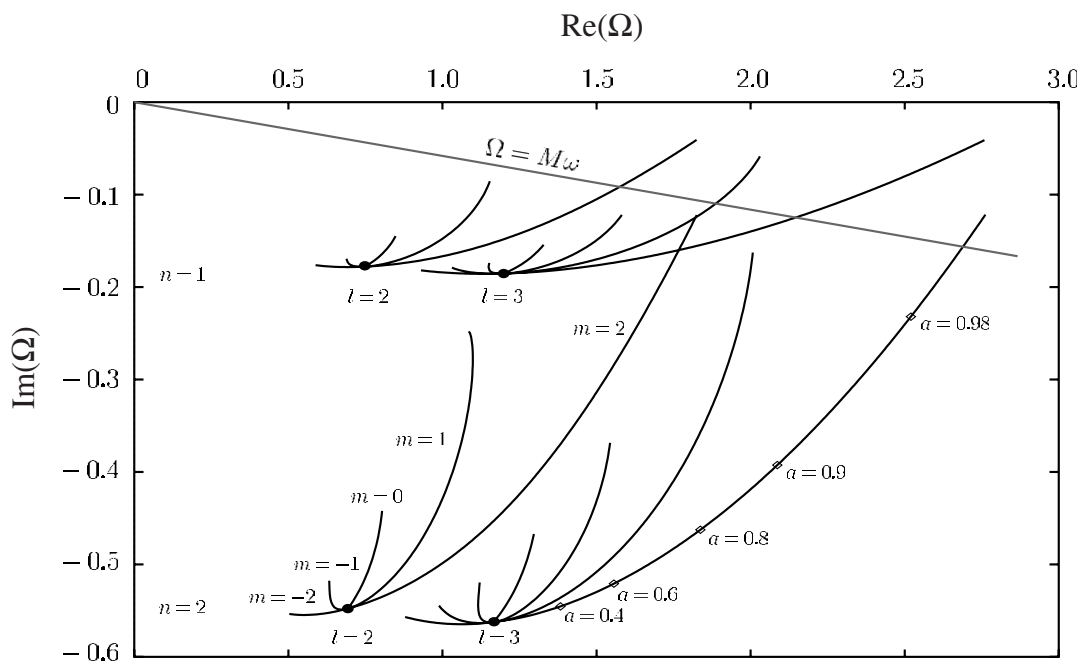

Figure 1. The dimensionless, complex QNM frequencies $\Omega_{n \ell m}$ for rotating, uncharged black holes. Each family of curves corresponds to one $n \ell$ pair, and each branch to a possible value of $m$. The large black dot at the base of each family is the Schwarzschild $(a=0)$ limit, where the frequencies are degenerate in $m$. This degeneracy is broken for $a \neq 0$, and the curves emanating from the dots give the QNM frequencies for Kerr black holes as a function of positive $a$ for different $m$. In this figure $a$ ranges from 0 to 0.9958 , with the small diamonds on the $\ell=3, m=3, n=2$ branch marking the QNM frequencies for $a=0.4,0.6,0.8,0.9$ and 0.98 . In this figure an observation, corresponding to a (complex) frequency $\omega$, is represented by the line $\Omega=M \omega$, parametrized by the (unknown) black-hole mass $M$. Each intersection of this line with a QNM curve in dimensionless $\Omega$ represents a candidate $n \ell m, M$ and $a$ for the mode.
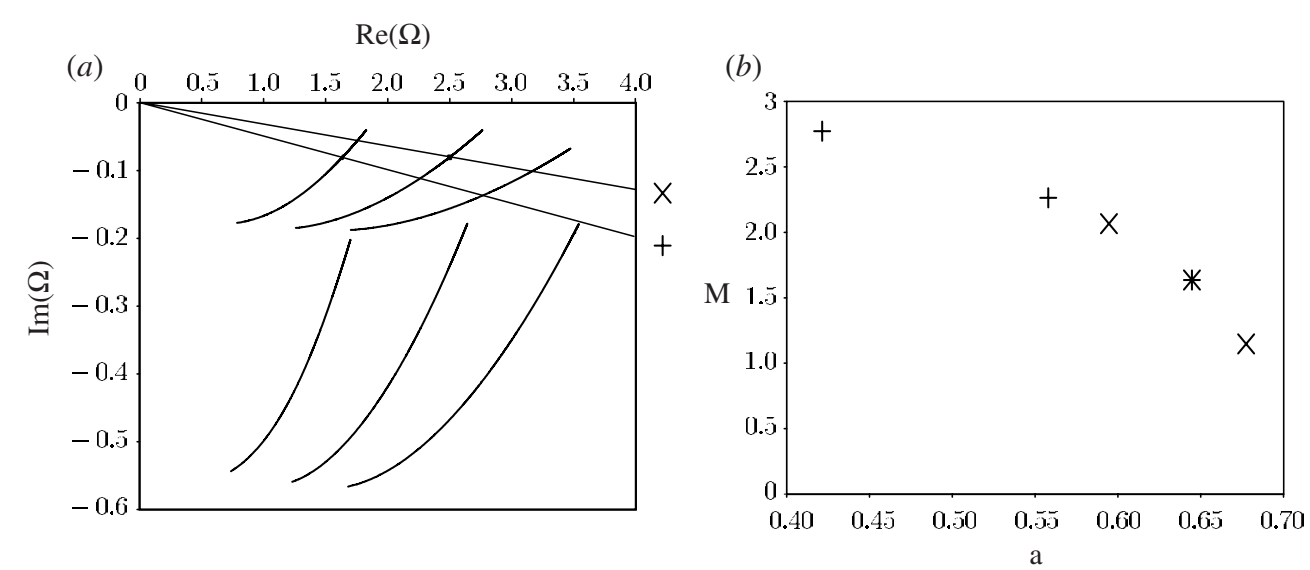

Figure 2. (a) Here we show, in schematic form, several $\Omega_{n \ell m}(a)$ curves and their intersection with the lines $M \omega_{i}, M>0, i=1,2$, corresponding to two observed modes. We denote these two lines by + and $\times$, respectively. $(b)$ The candidate $(a, M)$ pairs determined in $(a)$ are plotted here in the $(a, M)$-plane. The pairs belonging to $\omega_{1}$ are denoted by + , those belonging to $\omega_{2}$ by $\times$. There is only one candidate $(a, M)$ consistent with both observations, indicated by the overlapping + and $x$, and this is the actual mass and angular momentum of the underlying black hole.

curves in the complex $\Omega$ plane. Corresponding to each mode is a set of candidate $(a, M)$ pairs that may describe the underlying black hole. Each candidate mass and angular momentum 
parameter is a point in the $(a, M)$-plane, as shown in figure $2(b)$. With two or more modes, there must be at least one common candidate mass and angular momentum, indicated by the intersection of $\mathrm{a}+$ and $\mathrm{a} \times$.

This is, in essence, our proposed test: interpreting the observation of several normal modes $\omega_{k}, k \geqslant 2$, as arriving from a single, general relativistic black hole, and assuming that the no-hair theorem is true, then the observed $\omega_{k}$ will be consistent with at least one black hole $(a, M)$. If no such $(a, M)$ exists for the observed $\omega_{k}$ either we have observed something other than an isolated black hole or we have a contradiction with the predictions of the theory.

(As an aside, it is possible (though unlikely) that we get more than one value of $(a, M)$ consistent with the observed frequencies. This can happen if we have two mode pairs $\left(n_{1} \ell_{1} m_{1} ; n_{2} \ell_{2} m_{2}\right)$ and $\left(\tilde{n}_{1} \tilde{\ell}_{1} \tilde{m}_{1} ; \tilde{n}_{2} \tilde{\ell}_{2} \tilde{m}_{2}\right)$, which give rise to the same frequency $\omega$. In this case the observations would still be consistent with general relativity though we could not use that observation to measure $M$ and $a$. The important point of our test is the existence of at least one $(a, M)$ pair consistent with the observations.)

Noise and other experimental realities ensure that there will be no exact agreement between the observed $\omega_{k}$ and a general relativistic black hole even if general relativity is correct. The challenge, then, in developing a practical test is to determine when the differences between the candidate $(a, M)$ pairs associated with the different observed modes are so great as to be statistically inconsistent with general relativity. In the following section we face this challenge.

\section{A test of relativity}

\subsection{A reformulation of the test}

Before we discuss the role that noise plays in our analysis it is helpful to reformulate the test described in section 2.2 and figure 2. Consider an ordered $N$-tuple of QNMs,

$$
\mathcal{Q}:=\left\{\left(n_{k} \ell_{k} m_{k}\right): k=1, \ldots, N\right\} .
$$

This may be regarded as a function that maps a source $(a, M)$ to a set of observable frequencies

$$
\mathcal{Q}(a, M):=\left\{M^{-1} \Omega_{n_{k} \ell_{k} m_{k}}(a): k=1, \ldots, N\right\} .
$$

Each $N$-tuple $\mathcal{Q}$ thus describes a two-dimensional surface in the $(2 N+2)$-dimensional space $\mathcal{S}$,

$$
\mathcal{S}:=\left(a, M, \omega_{1}, \ldots, \omega_{N}\right)
$$

with different $N$-tuples corresponding to different sets of $N$ modes. (In section 3.3 we will understand the $\omega_{k}$ to represent observed QNM frequencies and damping times.)

An observation $\omega$ consists of an $N$-tuple

$$
\boldsymbol{\omega}:=\left(\omega_{1}, \ldots, \omega_{N}\right) .
$$

The observation $\boldsymbol{\omega}$ also corresponds to a surface in $\mathcal{S}$. The observation is consistent with a black hole if the surface of constant $\boldsymbol{\omega}$ intersects one of the surfaces $\mathcal{Q}$. Figure 3 shows a low-dimensional projection of such an observation $\omega$ together with several surfaces (which appear as curves) for different $N$-tuples $\mathcal{Q}$. A moment's consideration should convince one that this new criterion is equivalent to the test as described in section 2.2.

In practice the situation is less than ideal: noise distorts our observation, so that - even if we are observing black-hole QNMs - the measured $\omega$ will not intersect a surface $\mathcal{Q}$. In the remainder of this section we describe how this test is made practical and meaningful for real observations. 


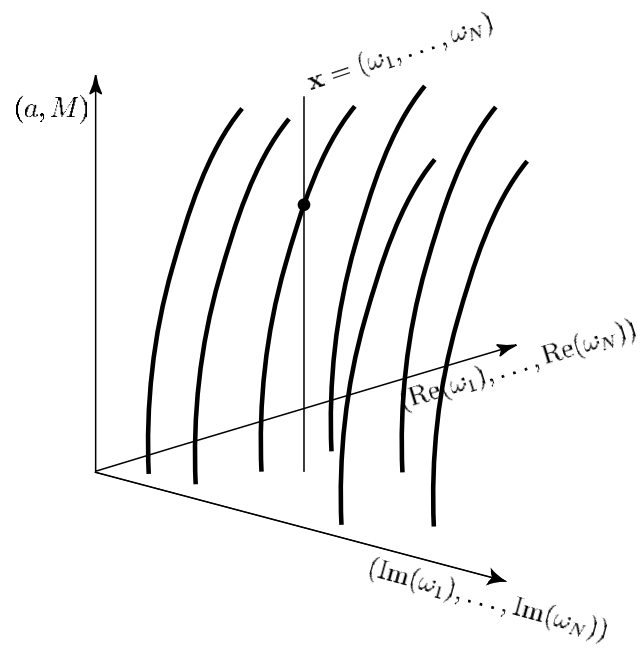

Figure 3. A reformulation of the consistency criterion. A set of quasi-normal modes $\mathcal{Q}=\left\{\left(n_{k} \ell_{k} m_{k}\right): k=1, \ldots, N\right\}$ corresponds to a surface in the $(2 N+2)$-dimensional space depicted in this figure. A measurement $\boldsymbol{\omega}=\left(\omega_{1}, \ldots, \omega_{N}\right)$ is consistent with general relativity if the constant surface that is obtained by ranging over all $(a, M)$ while keeping the frequencies $\omega$ fixed intersects at least one of the surfaces corresponding to one of the sets $\mathcal{Q}$. This intersection is indicated in this figure by a dot.

\subsection{Confidence intervals and testing general relativity}

In a frequentist analysis, the observation, the sampling distribution, an ordering principle and a probability combine to determine a confidence interval. In this section we use this construction to form a confidence region in the $(a, M)$-plane, given a noisy observation $\boldsymbol{\omega}$.

We begin by reviewing the construction of a classical confidence interval for the onedimensional case following [34] (alternatively, see, e.g., [35]). We suppose that we make measurements of a random variable $x$ from which a quantity $\mu$ is determined. The sampling distribution $P(x \mid \mu)$ is the probability of making the observation $x$ given a particular $\mu$. Formally, an ordering principle is a function $R(x \mid \mu)$, which we use to identify a sub-interval $J$ of $x$ according to

$$
J(\mu \mid r):=\{x: R(x \mid \mu)>r)\} .
$$

The parameter $r$ is chosen such that the region $J(\mu \mid r)$ encloses a fixed probability $p$ :

$$
\int_{J(\mu \mid r)} P(x \mid \mu) \mathrm{d} x=p .
$$

Given an observation $x_{0}$, the probability-p confidence interval $\mathcal{R}$ is the range of $\mu$ for which $J(\mu \mid r(p))$ includes $x_{0}$ as shown in figure 4. In an actual experiment, the choice of the value of the parameter $p$ is made by the experimentalist. Typical choices are $90 \%, 95 \%$ and $99 \%$.

The choice of the ordering principle $R(x \mid \mu)$ is a key ingredient in the construction of confidence intervals. Different choices will lead to different confidence intervals for the same observation: for example, one choice of ordering principle will always determine intervals of the form $(-\infty, x)$, while another choice will always determine intervals of the form $(x, \infty)$. Neither choice is a priori right or wrong. Here we will choose $R(x \mid \mu)=P(x \mid \mu)$ so that the intervals are given by level surfaces of the distribution $P(x \mid \mu)$. The main advantage of this ordering principle is that it is simple and it works in any dimension. Consider, for example, 


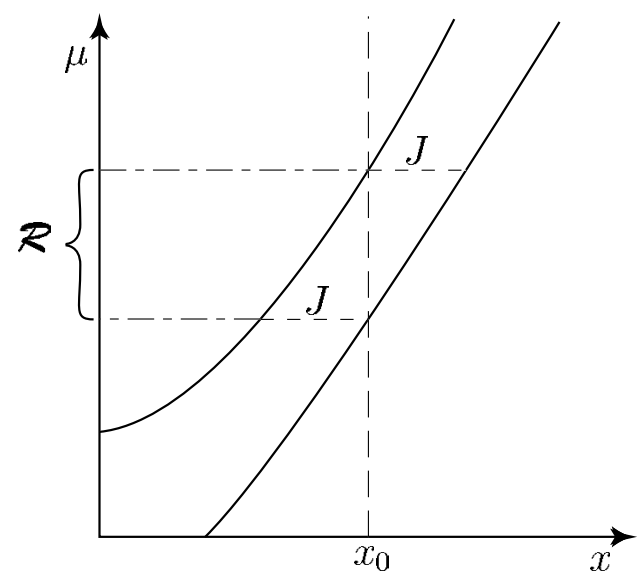

Figure 4. The construction of classical confidence intervals. A sampling distribution $P(x \mid \mu)$, an ordering principle $R$ and a probability $p$ are needed to construct a confidence interval. The ordering principle is used to find the intervals $J(\mu)$ such that $\int_{J(\mu)} \mathrm{d} x P(x \mid \mu)=p$. The classical confidence interval $\mathcal{R}$ is then given by the set of $\mu$ for which $J(\mu)$ contains the measured value $x_{0}$.

a two-component observation depending on one parameter $a$. There is, as before, a sampling distribution $P(x, y \mid a)$ and an ordering principle $R(x, y \mid a)=P(x, y \mid a)$. Confidence intervals can be defined in the same way as in the case of a one-dimensional observation; the interval $J$ is now a two-dimensional region. Since this system is over-determined-we are now trying to determine one parameter $a$ by measuring two quantities $x$ and $y$-the measured $x$ and $y$ will have to satisfy additional constraints in order to give a non-vanishing confidence region. This is in fact precisely what happens in the black-hole quasi-normal mode problem: any single measurement of $\omega$ can be explained by some $(a, M)$, but a measurement of two or more $\omega$ can be simultaneously consistent with at least one $(a, M)$ pair only if the no-hair theorem is true and the modes arise from a single black hole.

We can now describe our test of relativity: note that some observations $\boldsymbol{\omega}$ will lead to an empty confidence interval; i.e., for some $\boldsymbol{\omega}$ there will be no $(a, M)$ consistent with the observation. If we make an observation $\boldsymbol{\omega}$ for which the probability- $p$ confidence interval is empty, then we say that the observed normal modes are inconsistent with an isolated black hole with confidence $p$. Conversely, if there does exist a non-empty probability- $p$ confidence interval, then we have verified that general relativity is self-consistent at this confidence level.

Finally, we should point out an aesthetic flaw of our choice of ordering principle. The function $P(x \mid \mu)$ is a density and, therefore, not invariant under a reparametrization of $x$. If we were to use a new parameter $x^{\prime}=f(x)$ for some smooth monotonic function $f$, the confidence region obtained for $\mu$ using a measurement of $x$ may not coincide with the region obtained using a measurement of $x^{\prime}$. In the one-dimensional case, there exists another ordering principle based on the likelihood ratio [36] which is reparametrization invariant; however, we have not been able to generalize this to higher dimensions. While aesthetically displeasing, there is nothing wrong with the choice we have made, which is natural given the physical association of the parameters $M$ and $a$ with the black-hole mass and angular momentum.

\subsection{Generalization to quasi-normal modes}

The generalization to QNM observations is straightforward. Each observation consists of $N$ complex QNM frequencies $\omega_{k}$ and associated amplitude signal-to-noise ratios $\rho_{k}$, which 


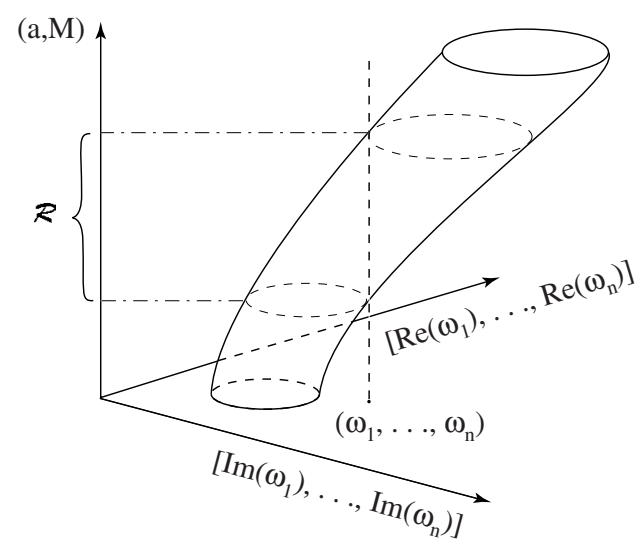

Figure 5. The construction of classical confidence intervals generalized to higher dimensions. Given a sampling distribution $P$, an ordering principle $R$ and a probability $p$ one can construct classical confidence regions $\mathcal{R}$ just as in the one-dimensional case. The difference here is that we are now trying to determine a small number of parameters $(a, M)$ from a larger number of observations $\boldsymbol{\omega}=\left(\omega_{1}, \ldots, \omega_{N}\right)$. There are thus additional consistency conditions that need to be satisfied to obtain a non-empty confidence region $\mathcal{R}$.

characterize both the amplitude of the signal at that frequency and the uncertainty in the determination of $\omega_{k}(\mathrm{cf}[5])$ :

$$
\begin{aligned}
\boldsymbol{\omega} & :=\left(\omega_{1}, \ldots, \omega_{N}\right) \\
\boldsymbol{\rho} & :=\left(\rho_{1}, \ldots, \rho_{N}\right) .
\end{aligned}
$$

For definiteness suppose that $\omega_{k}$ and $\rho_{k}$ are identified via maximum likelihood techniques [5]. There is a minimum signal-to-noise associated with each mode, which is set by the requirement that the observation must identify $N$ modes.

Observations $\boldsymbol{\omega}$ corresponding to a black hole characterized by $(a, M)$ and signals-tonoise $\rho$ are distributed according to the sampling distribution

$$
P(\omega \mid a, M, \mathcal{Q}, \rho):=\left(\begin{array}{c}
\text { Probability of making observation } \\
\omega \text { given the actual } N \text {-tuple } \mathcal{Q} \\
\text { and signals-to-noise } \rho .
\end{array}\right) .
$$

In general, the sampling distribution depends upon the nature of the detector noise and the analysis procedure that identifies the modes $\omega_{k}$. For large signal-to-noise ratios it will generally reduce to a multivariate Gaussian in $\operatorname{Re}\left(\omega_{k}\right)$ and $\operatorname{Im}\left(\omega_{k}\right)$ and for smaller signal-to-noise ratios it can be determined via simulation.

Now consider the region of the space $\mathcal{S}$ (cf section 3.1) defined by

$$
P(\boldsymbol{\omega} \mid a, M, \mathcal{Q}, \boldsymbol{\rho})>p_{0}
$$

with $p_{0}$ such that

$$
\int_{P(\omega \mid a, M, \mathcal{Q}, \rho)>p_{0}} P(\boldsymbol{\omega} \mid a, M, \mathcal{Q}, \boldsymbol{\rho}) \mathrm{d}^{2 N} \omega=p
$$

for a fixed $p$. We say that the observation $\omega$ is consistent with a black hole if the actual observation $\boldsymbol{\omega}$ is included in this region for some $(a, M)$. Figure 5 illustrates the comparison of an observation with the region defined by equations (13), (14). 
To help in specifying $p$ it is useful to examine its meaning more closely. Suppose we have chosen a value of $p$. That value determines a confidence region. Now consider an ensemble of identical detectors, each observing simultaneously the same black-hole event and its corresponding QNMs. The fraction of these observations that does not intersect the confidence region is the false alarm probability $\alpha(p)$, so-called because it is the probability that an observation will be falsely deemed to be inconsistent with a black hole. The probability $\alpha$ is a monotonic function of $p$; therefore, we can specify $\alpha$ in lieu of $p$. For observations that we are confident originate with black holes (because their characteristic frequency corresponds to masses greater than neutron star masses), we propose setting $p$ so that $\alpha(p)$ - the probability of falsely rejecting the hypothesis that we have in fact observed a black hole-is small (e.g., less than 1\%). In other words, the standard of evidence for declaring that we have discovered 'new physics' should be high.

The false alarm probability function $\alpha(p)$ will depend on the signal strength, as characterized by the signal-to-noise ratios; consequently, it will need to be determined on an observation-by-observation basis. Thus the calculation of $\alpha(p)$ by a Monte Carlo simulation is the final ingredient we need. In the following section we demonstrate the test through a numerical example where we calculate $\alpha(p)$.

\section{A numerical example}

In the previous section we described a general procedure for testing general relativity by observing QNMs. In this section we explore its effectiveness numerically through a set of simulated observations drawn from a hypothetical black-hole population inspired by potential LISA observations, and a hypothetical population of non-black-hole compact object sources, or NBHs. (We say 'inspired' because, in fact, for the purpose of this analysis the observations are characterized entirely by the dimensionless signal-to-noise ratio and mode quality factor, with the dimensioned mode frequency simply setting a scale. Thus, the conclusions we reach are as valid for LISA observations as they are for observations at the same signal-to-noise with ground-based detectors.)

For the black-hole $(\mathrm{BH})$ observations we find the relationship between the false alarm probability $\alpha$ and the probability $p$ that appears in equation (14). For the NBH observations there are no 'false alarms': every observation is of something not a black hole. Instead, there are false dismissals: observations that we mistakenly classify as consistent with a black hole. The probability of a false dismissal, denoted by $\beta$, depends on the choice of $p$ or, alternatively, the choice of false alarm probability $\alpha(p)$ that we make for the purpose of defining the test. (The false dismissal probability depends also on how the spectra of BHs and NBHs differ.) The smaller the false dismissal probability the more sensitive the test is to discovering 'new physics' or identifying non-black-hole sources. For the NBH observations we evaluate the false dismissal probability as a function of the false alarm probability.

\subsection{Mode detection}

Our concern here is with the question of statistical inference from QNM observations, characterized by their signal-to-noise, frequency and damping time. We do not venture to explore how, from a signal-processing standpoint, these events are identified and so characterized and none of the qualitative conclusions drawn in this section depend on the method of event identification and characterization. Nevertheless, it is worthwhile to comment briefly on the challenges associated with detecting and characterizing damped sinusoidal signals. 
Matched filtering is often invoked as the preferred method for detecting signals in noise when the signal is known exactly, up to a few parameters. This does not mean, however, that matched filtering is the most computationally efficient means of detection. Similarly, while matched filtering may be optimal for these problems, it does not mean that there is necessarily a large difference in efficiency between matched filtering and other less optimal methods of event identification. Matched filtering stands-out only when the signal being sought has significant defining features that allow it to be discriminated from noise events. This is not the case for damped sinusoids with low quality factor $Q$.

In fact, matched filtering is known to be a very poor way to search for damped sinusoids. A matched filter search for a damped sinusoid of unknown frequency and damping time in a time-series $h(t)$ is equivalent to taking the Laplace transform of $h(t)$, which is computationally difficult to do accurately. (A set of 'matched filter templates', consisting of damped sinusoids, applied to a time-series $h(t)$ corresponds to a sample of the Laplace transform of $h$.) Fortunately, the problem of analysing noisy data for damped sinusoidal signals is not unique to gravitational-wave physics and other methods, beyond matched filtering, exist (cf, e.g., [37, 38]).

While none of the results below depend on the method of detection, we chose, where choices need to be made, to use theoretical results from matched filtering studies to describe the uncertainties associated with frequency and damping time determination. Such choices do not affect our qualitative conclusions and, in any event, relevant quantitative conclusions could not be drawn without both LISA data and the choice of a specific analysis method.

\subsection{Simulating black-hole QNM observations}

For definiteness we focus on observations of two QNMs (this corresponds to $N=2$ in section 3). For the purpose of illustration we consider black-hole masses and angular momenta consistent with potential observations by the LISA detector [7]. We first draw an $(a, M)$ pair from the distribution

$$
\begin{aligned}
& P(a, M)=P(a) P(M) \\
& P(a) \propto \begin{cases}1 & \text { for } a \in[0,0.986) \\
0 & \text { otherwise }\end{cases} \\
& P(M) \propto \begin{cases}M^{-1} & \text { for } M \in\left(2.5 \times 10^{5} M_{\odot}, 4.5 \times 10^{8} M_{\odot}\right) \\
0 & \text { otherwise. }\end{cases}
\end{aligned}
$$

The range of $M$ is determined by the frequency band where LISA is expected to be most sensitive; the range of $a$ is determined by the maximum angular momentum expected of a black-hole spun-up by thin-disc accretion [39].

Corresponding to each $(a, M)$ pair we choose the QNMs corresponding to $(n=1, \ell=$ $2, m=2)$ and $(n=1, \ell=4, m=4)$. We assign each mode the same signal-to-noise ratio, which we treat here as sufficiently large that the errors associated with the measurements are normally distributed with covariance matrix $C_{i j}$ equal to the inverse of the Fisher information matrix $I_{i j}$ (see, e.g., [35]) as given in [5, equation (4.14)]. This is in fact a mathematical lower bound-the Cramer-Rao bound - on the covariance matrix. We draw from this error distribution errors in the frequencies and damping times that we add to the 'real' frequencies and damping times to determine the simulated observations: noisy QNM frequencies and damping times.

Given this pair of QNM frequencies and damping times with errors we ask whether the two modes are in fact observationally distinguishable: if the frequencies and damping times 
are not sufficiently different, then no real observation would ever result in the given pair. For instance, the five $(n=1, l=2)$ modes are degenerate at $a=0$; consequently, no matter how large the signal-to-noise ratio, if $a$ is sufficiently small it is impossible to resolve these five modes observationally.

To decide whether the two modes we investigate are observationally distinguishable we invoke a 'resolvability criterion': denoting the frequencies (damping times) of the two modes as $f_{1}, f_{2}\left(\tau_{1}, \tau_{2}\right)$ we say that the two modes are distinguishable if

$$
\left|f_{1}-f_{2}\right|>\frac{1}{\min \left(\tau_{1}, \tau_{2}\right)} \text {. }
$$

We discard any mode pair that does not satisfy this criterion.

The result of this procedure is an observation, which consists of a pair of signal-to-noise ratios and associated distinguishable frequencies and damping times. (The observation does not include knowledge of the black-hole mass or angular momentum, or the $n \ell m$ associated with the frequencies or damping times.)

\subsection{False alarm probability $\alpha$}

For each simulated observation $\omega$, constructed as described in section 4.2 , we evaluate the smallest probability $p=p_{\min }$ such that equations (13) and (14) describe a region $\mathcal{S}$ that covers $\boldsymbol{\omega}$ for some $(a, M)$. The false dismissal fraction $\alpha(p)$ is the fraction of $p_{\text {min }}$ determinations that are greater than $p$, i.e., the fraction of $\mathrm{BH}$ observations that we would reject as originating from a black hole for threshold $p$.

Ideally, in evaluating $p$ we would consider every possible $n \ell m$ for each $\omega_{k}$. In practice, we consider only a finite subset of low-order (in both $n$ and $\ell$ ) modes, corresponding to our expectation that these are the modes most likely to be excited to large amplitude. In our simulations we considered only modes corresponding to $(n=1, \ell=2, m=0),(n=1, \ell=$ $2, m=2),(n=1, \ell=3, m=3)$ and $(n=1, \ell=4, m=4)$. Since for these simulations we observed two distinguishable QNMs there were 12 possible ordered pairs of modes. Figure 6 shows $\alpha$ as a function of $p$ for four different signal-to-noise ratios. Each $\alpha(p)$ curve is constructed from $10^{4}$ simulated observations with that amplitude-squared signal-to-noise in each mode.

\subsection{False dismissal probability calculation}

Complementary to $\alpha$, the probability that we incorrectly decide we have observed QNMs from something other than a black hole, is the probability that we falsely conclude we have observed QNMs from a black hole. This probability is referred to as the false dismissal probability and commonly denoted as $\beta$.

The false dismissal probability depends on the detailed character of the source, which is not a black hole. Strong gravitational-wave sources are compact, with radius $R$ not much greater than their mass $G M / c^{2}$ and oscillation periods of order $G M / c^{3}$. At the frequencies where LISA will have its greatest sensitivity, $10^{-2}-10^{-4} \mathrm{~Hz}$, corresponding to masses of order $10^{6}-10^{8} M_{\odot}-$ we know of no compact sources that are not black holes. For the purpose of illustration and to give a sense of the ability of the test described here to 'discover' new physics, we suppose a population of sources whose frequencies and damping times share the same relationship as certain neutron star w-modes calculated in [8]. Referring to [8, table 1, column 1 , lines 3,5 ] we consider observations consisting of two modes

$$
M \omega_{1}=0.471+0.056 \mathrm{i}, \quad M \omega_{2}=0.654+0.164 \mathrm{i}
$$




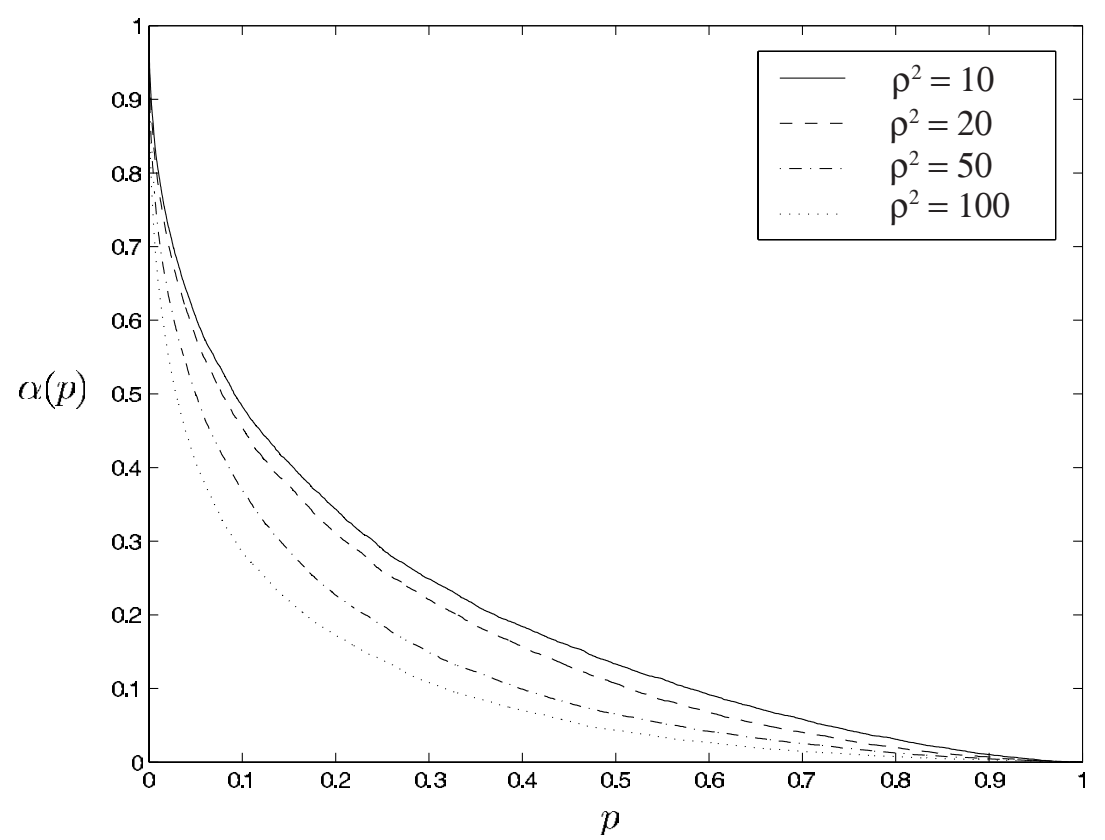

Figure 6. False alarm probability $\alpha$ as a function of the probability $p$ appearing in equation (14). A false alarm is a misidentification of a QNM pair as arising from something other than a general relativistic black hole.

where $M$ is drawn from the distribution given in equation (17). In exactly the same way that we used simulations in section 4.3 to determine $\alpha$ as a function of $p$ we calculate from these simulations $\beta$ as a function of $p$. Together $\alpha(p)$ and $\beta(p)$ determine $\beta(\alpha)$, which we show in figure 7. A measure of the effectiveness of the test is the degree to which the curves for different signal-to-noise fall below the $\beta=1-\alpha$ diagonal. (A 'test' that randomly picked a fraction $\alpha$ of observations as not black holes would have $\beta=1-\alpha$. Any 'test' that can do better than randomly choosing in this way will have a $\beta(\alpha)$ curve that falls below this diagonal.) As expected the test also does better with stronger signals. Consider a false alarm threshold of $1 \%$. Then for observations with $\rho^{2}=10$ we have a better-than- $40 \%$ chance of distinguishing NBH sources from BH sources. This climbs to better-than- $90 \%$ chance for observations with $\rho^{2}=100$.

\section{Potential for application}

We have shown that, given at least two QNM signals from the same source and with sufficiently large signal-to-noise we can clearly distinguish black holes from other astrophysical sources. We have also demonstrated how this can be used as a test of general relativity. In this section, we investigate the potential for application of this test in future LISA observations by asking, first,

- How distant can LISA-scale black-hole sources be and still have multiple QNMs detected at sufficiently high signal-to-noise?

and, secondly,

- What is the rate of sources that we may expect within this distance? 


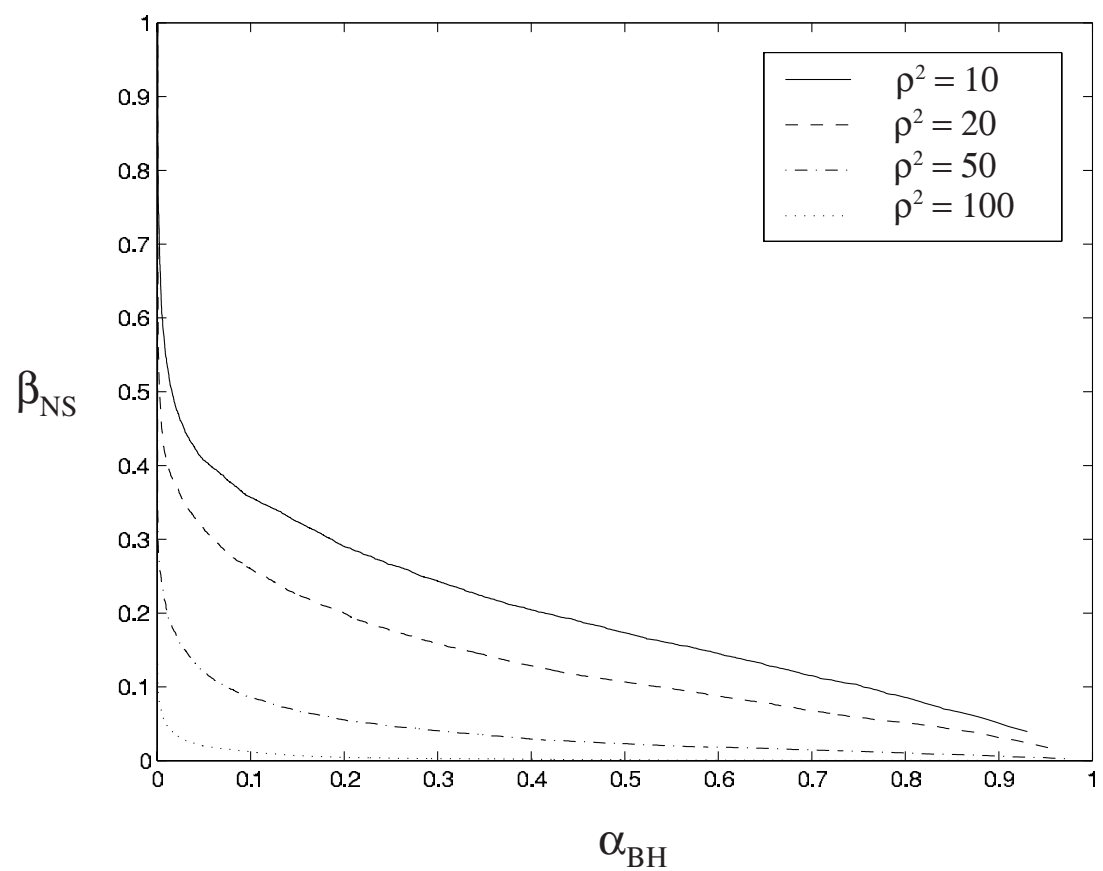

Figure 7. False dismissal probability as a function of false alarm probability $\beta(\alpha)$. The false dismissal probability depends on the non-black-hole QNM spectrum, which we have taken to have the same ratio of frequencies and relationship between frequencies and damping times as neutron star w-modes.

To begin we evaluate, as a function of distance and energy radiated in a QNM, the signalto-noise in the LISA detector. We focus attention on an individual QNM. The signal strength, characterized by the signal-to-noise ratio at the detector, depends on the energy radiated in the mode the radiation pattern associated with the mode and the relative orientation of the detector and the source. Following [40, equation (2.30)] we can average over these latter angles to obtain the mean-square signal-to-noise associated with the $n \ell m$ mode as a function of the mode energy

$$
\left\langle\rho^{2}\right\rangle=\frac{2(1+z)^{2}}{5 \pi^{2} D(z)^{2}} \int_{0}^{\infty} \mathrm{d} f \frac{1}{f^{2} S_{n}(f)} \frac{\mathrm{d} E_{e}}{\mathrm{~d} f_{e}}[(1+z) f]
$$

where $z$ and $D(z)$ are, respectively, the redshift and the luminosity distance to the source.

For the mode, we assume the form

$$
h_{n \ell m}(t)=A_{n \ell m} \exp \left(-\frac{\pi f_{n \ell m} t}{Q_{n \ell m}}\right) \sin \left(2 \pi f_{n \ell m} t\right)
$$

where $Q_{n \ell m} \equiv \pi f_{n \ell m} \tau_{n \ell m}$. Note that $Q_{n \ell m}$, which is an observable property of a QNM, is independent of source redshift, while the observed $f_{n \ell m}$ and $\tau_{n \ell m}$ depend on redshift.

The ringdown energy spectrum of the $n \ell m$ mode is taken from equation (3.18) of [40]

$$
\frac{\mathrm{d} E_{e}}{\mathrm{~d} f_{e}}=\frac{\epsilon_{n \ell m}}{F_{n \ell m}} \frac{Q_{n \ell m}}{\left(4 Q_{n \ell m}^{2}+1\right)} \frac{M^{2} f^{2}}{\pi^{3} \tau^{2}}\left[\frac{1}{\left[\left(f-f_{n \ell m}\right)^{2}+(2 \pi \tau)^{-2}\right]^{2}}+\frac{1}{\left[\left(f+f_{n \ell m}\right)^{2}+(2 \pi \tau)^{-2}\right]^{2}}\right],
$$


where the mode amplitude $A_{n \ell m}$ has been replaced with the fraction $\epsilon_{n \ell m}$ of the mass radiated in that mode, defined by

$$
\epsilon_{n \ell m}:=\frac{1}{M} \int_{0}^{\infty} \frac{\mathrm{d} E}{\mathrm{~d} f} \mathrm{~d} f
$$

Using this spectrum in the formula above, and approximating the LISA noise power spectral density $S_{n}(f)$ as constant over the signal band, we integrate over frequencies and invert the result to obtain an approximate distance to which we can observe a mode $n \ell m$ with signal-tonoise greater than $\rho_{n \ell m}^{2}$ :

$$
D(z)^{2}<\frac{8}{5 \pi^{2}} \frac{Q_{n \ell m}^{2}}{4 Q_{n \ell m}^{2}+1} \frac{(1+z)^{3} M^{3}}{F_{n \ell m}^{2}} \frac{\epsilon_{n \ell m}}{S_{n} \rho_{n \ell m}^{2}} \frac{G^{3}}{c^{7}} .
$$

The relationship between luminosity distance and redshift we take to be given by equations (23), (25) of [44]. This relationship depends on cosmological parameters that characterize the universe and its expansion and for these we use the values determined by the first season's WMAP observations [45]. Thus, given a threshold $\rho_{n \ell m}^{2}$, black holes radiating a fraction $\epsilon_{n \ell m}$ of their rest energy in mode $n \ell m$ are observable within a redshift $z$ satisfying equation (24).

Numerical simulations suggest that the energy emitted in QNMs during ringdown may be of order $1 \%$ of the rest-mass energy of the hole [41, 42]. For equal-mass black-hole mergers, the simulations suggest that the $\ell=2$ modes will be by far the strongest with total emitted energies greater than the $\ell=4$ modes by as much as three orders of magnitude (see [43]). Thus we may assume that the weaker mode of a QNM pair produced immediately following an equal mass black-hole merger carries away a fraction $10^{-5}$ of the final black-hole mass. To be more conservative, we consider instead a considerably smaller emitted mass fraction $\epsilon_{n \ell m}=10^{-7}$.

LISA will be most sensitive in the frequency band $10^{-3} \leqslant f \leqslant 10^{-2} \mathrm{~Hz}$ where the noise power spectral density is expected to be $5 \times 10^{-45} \mathrm{~Hz}^{-1}$. Black holes at redshift $z$ whose (low-order) QNM frequencies peak in this band have a mass of order $\mathcal{M} /(1+z)$, where $\mathcal{M}$ ranges from $10^{6}$ to $10^{7} M_{\odot}$. Focusing just on these black holes we find, from equation (24), that LISA can expect to see pairs of QNMs associated with black-hole mergers, with the weaker mode having a signal-to-noise $\rho>10$, within a redshift of $\sim 52$ (for extremal-spin Kerr) or $\sim 36$ (for Schwarzschild); that is, LISA will be able to observe mergers associated with the assembly of essentially all galaxies throughout the universe. Considering a broader frequency band of $10^{-1}-10^{-4} \mathrm{~Hz}$ will increase the mass range (and thus rate of observed mergers) further with the addition of more and less massive (redshifted) black-hole mergers, though to a somewhat smaller redshift.

To summarize, we expect that pairs of QNMs associated with the mergers of supermassive black holes in the redshifted mass range of $10^{6}-10^{7} M_{\odot}$ will be observable by LISA with significant signal-to-noise throughout the observable universe as long as the weaker mode carries away at least a fraction $10^{-7}$ of the final black-hole mass. The rate of such black-hole mergers depends on redshift owing to evolution and the scaling of the intrinsic black-hole mass corresponding to observed QNM frequency with redshift. There is considerable uncertainty at present about the event rate for such observations; however, estimates out to $z=20$ - far smaller than the range within which LISA is sensitive-range from 0.3 to 100 per year [46-48]. Correspondingly, we expect that this test will find application in forthcoming LISA observations. 


\section{Conclusion}

We have described a qualitatively new test for the existence of general relativistic black holes, based on the gravitational radiation they emit when they are formed or when they are impulsively excited, for example, through a merger event. Radiation from an impulsively excited black hole, such as might arise in the course of a non-spherical black-hole formation event or the coalescence of a black hole with another black hole or compact object, has a component that consists of a sum of damped sinusoids. This signature is characteristic of the radiation from any impulsively excited, damped source. For any given mode, the scale of the frequency and damping time measures the black-hole mass and angular momentum. Similarly, the relationship of the different modes to each other-i.e., the spectrum-is unique to black holes. We have described here how this relationship can be used to test the proposition that observed gravitational waves, characteristic of an impulsively excited, damped source, in fact originate from a general relativistic black hole. Such a test can be characterized in at least two different ways: as a definitive 'proof' that a black hole has been observed or as a test of the so-called 'no-hair' theorem of general relativity.

To demonstrate the effectiveness of this test we have evaluated numerically the probability that the test will mistakenly fail to identify an actual black hole. By introducing a hypothetical gravitational-wave source whose characteristic frequencies and damping times are similar to those of neutron star w-modes [8], we have also evaluated numerically the probability that the test will incorrectly identify w-mode oscillations of a neutron star, or any object whose spectrum is similar to that of a black hole. Together these results demonstrate that for sources with the signal-to-noise expected of, for example, massive black-hole coalescences detected by LISA, the test proposed here can clearly discriminate black-hole sources. Finally, we have shown that LISA can be expected to observe black-hole ringdown signals of this kind and strength almost throughout the observable universe. However, the event rates for such detections are rather uncertain and further work is needed to ascertain whether this test will be applicable in practice.

This method can also be used to measure mass and angular momentum of a black hole. Using gravitational waves to measure mass and angular momentum is an idea that has been around for some time $[4,5]$. In these previous works it was assumed that the mode observed was of a known order (e.g., the mode with the longest damping time or the lowest order, etc.). With the observation of two or more modes the requirement that a single mass and angular momentum explain the complete set likely permits the mass and angular momentum to be determined uniquely.

The field of gravitational-wave detection is new. The current generation of ground- and space-based gravitational-wave detectors is opening a new frontier of physics: gravitationalwave phenomenology, or the use of gravitational-wave observations to learn about the physics of gravitational-wave sources and gravity itself. We are only just beginning to learn how to exploit the opportunities it is creating for us. As gravitational-wave observations mature, we can expect more and greater recognition of their utility as probes of the character of relativistic gravity. The opening of this new frontier promises to be an exciting and revealing one for the physics of gravity.

\section{Acknowledgments}

We are grateful to Soumya Mohanty and Curt Cutler for helpful discussions. We acknowledge support through the Center for Gravitational Wave Physics, which is funded by the NSF under 
cooperative agreement PHY 01-14375, NSF awards PHY 00-90091 and PHY 00-99559, the Eberly research funds of Penn State and the Albert-Einstein Institut.

\section{References}

[1] Cunningham C T et al 1978 Astrophys. J. 224 643-67

[2] Cunningham C T et al 1979 Astrophys. J. 230 870-92

[3] Cunningham C T et al 1980 Astrophys. J. 236 674-92

[4] Echeverria E 1989 Phys. Rev. D 40 3194-203

[5] Finn L S 1992 Phys. Rev. D 46 5236-49

[6] Danzmann K et al 1998 LISA - laser interferometer space antenna, Pre-Phase A Report Max Planck Institut für Quantum-Optiks, Report MPQ 233

[7] Finn L S and Thorne K S 2000 Phys. Rev. D 62124021

[8] Andersson N et al 1995 Mon. Not. R. Astron. Soc. 274 1039-48

[9] Seidel E and Suen W 1990 Phys. Rev. D 42 384-403

[10] Alcock C et al 1986 Astrophys. J. 310 261-72

[11] Gibbons G W and Maeda K 1988 Nucl. Phys. B 298 741-75

[12] Eardley D M et al 1973 Phys. Rev. D 8 3308-21

[13] Taylor J H and Weisberg J M 1982 Astrophys. J. 253 908-20

[14] Finn L S 1985 Class. Quantum Grav. 2 381-402

[15] Ryan F D 1997 Phys. Rev. D 56 1845-55

[16] Will C M 1998 Phys. Rev. D 57 2061-8

[17] Finn L S and Sutton P J 2002 Phys. Rev. D 65044022

[18] Sutton P J and Finn L S 2002 Class. Quantum Grav. $191355-60$

[19] Scharre P D and Will C M 2002 Phys. Rev. D 65042002

[20] Fairhurst $\mathrm{S}$ et al in preparation

[21] Leaver E W 1985 Proc. R. Soc. Lond. A 402 285-98

[22] Onozowa H 1997 Phys. Rev. D 55 3593-602

[23] Nollert H P 1999 Class. Quantum Grav. 16 R159-216

[24] Kokkotas K D and Schmidt B G 1999 Liv. Rev. Rel. 2 http://www.livingreviews.org/lrr-1999-2

[25] Berti E and Kokkotas K D 2003 Phys. Rev. D 68044027

[26] Berti E et al 2003 Preprint hep-th/0307013

[27] Regge T and Wheeler J A 1957 Phys. Rev. 108 1063-9

[28] Zerilli F 1970 Phys. Rev. Lett. 24 737-8

[29] Zerilli F J 1971 Phys. Rev. D 2 2141-60

[30] Vishveshwara C V 1970 Nature 227 936-38

[31] Teukolsky S A 1972 Phys. Rev. Lett. 29 1114-8

[32] Teukolsky S A 1973 Astrophys. J. 185 635-47

[33] Press W H and Teukolsky S A 1973 Astrophys. J. 185 649-73

[34] Neyman J 1937 Phil. Trans. R. Soc. Lond. A 236 333-80

[35] Stuart A and Ord J K 1994 Kendall's Advanced Theory of Statistics vol 1 (London: Edward Arnold)

[36] Feldman G J and Cousins R D 1998 Phys. Rev. D 57 3873-89

[37] Hamming Richard 1987 Numerial Methods for Scientists and Engineers (New York: Dover)

[38] Shahram M and Milanfar P 2003 On the resolvability of multiple sinusoids with nearby frequencies in the presence of noise Preprint (IEEE Trans. Signal Process. at press)

[39] Thorne K S 1974 Astrophys. J. 191 507-19

[40] Flanagan É É and Hughes S A 1998 Phys. Rev. D $574535-65$

[41] Brandt S and Seidel E 1995 Phys. Rev. D 52 870-86

[42] Khanna G et al 1999 Phys. Rev. Lett. 83 3581-4

[43] Anninos P et al 1995 Phys. Rev. D 52 4462-80

[44] Carroll S M and Press W H 1992 Annu. Rev. Astron. Astrophys. 30 499-542

[45] Spergel D N et al 2003 Astrophys. J. Suppl. 148 175-94

[46] Haehnelt M G 2003 Class. Quantum Grav. 20 S31-6

[47] Haehnelt M G 2003 Joint formation of supermassive black holes and galaxies Carnegie Observatories Astrophysics Series Vol 1: Co-evolution of Black Holes and Galaxies ed L C Ho (Cambridge: Cambridge University Press)

[48] Cutler C and Thorne K S 2002 Preprint gr-qc/0204090 